\title{
A Static Low Energy Ion Source for Local Surface Modification
}

\author{
Johannes J L Mulders ${ }^{1}$, Petrus HF Trompenaars ${ }^{1}$ \\ 1. FEI Company, Achtseweg Noord 5, 5600KA Eindhoven, The Netherlands
}

A new in-situ low energy ion source for SEM and DualBeam has been designed. The static beam of low energy gaseous ions such as $\mathrm{Ar}^{+}, \mathrm{O}^{+}$or $\mathrm{Xe}^{+}$can be used for a local modification of the sample surface. Typical energies are in the range $5-500 \mathrm{~V}$, covering the interaction types from chemical reaction to reactive ion etching and to ion milling, for energies above the milling threshold. The source is based on the following principle: electrons from the SEM's electron beam partially convert an atomic or molecular gas flow into a beam of ions directed towards a biased sample. A schematic set up is shown in figure 1. A small nozzle delivers the gas and the electron beam enters this nozzle through a slotted hole. The beam is scanned in this slotted hole, penetrates the gas flow and generates thermal ions both by direct ionization and by ionization from beam interactions with the wall of the nozzle. The ions are pulled out of the nozzle by the protruding fields from the biased sample which is located at a short distance from the nozzle: the ions are accelerated in this electrostatic field and directed towards the sample. The slotted entry hole is roughly located at half the inner nozzle diameter from the edge.

For quantitative determination of the beam profile of the source, use is made of the sputtering capability of the ions. The local ion flux on the sample is linearly related to the amount of material that is milled away. To this end, a silicon sample has been coated with Pt of high purity using an atomic layer deposition (ALD) process and the thickness is calibrated against an ellipsometer. The normalized EDX net peak intensity ratio of PtM and $(\mathrm{SiK}+\mathrm{PtM})$ are measured and plotted against the real thickness (Figure 2). In this way the local Pt layer thickness is determined with an accuracy of $\sim 1 \mathrm{~nm}$ and lateral resolution < $30 \mathrm{~nm}$. Using a $23 \mathrm{~nm}$ Pt film on Si and exposing this to the low energy ion beam, the local thickness can be determined with automated collection of EDX spectra at various points of the beam impact area. The local thickness profile corresponds to the ion beam profile, such as shown in Figure 3.

The source produces a static beam of ions with selectable energy. The direction and width of the beam depend on the geometry and not on the applied bias voltage, because the electric fields define both the acceleration and the trajectories. With a typical SEM excitation condition of $2 \mathrm{kV}$ and $26 \mathrm{nA}$ it is possible to generate a $100 \mathrm{eV} \mathrm{Ar}{ }^{+}$beam current of $5 \mathrm{nA}$ and a FWHM of $8.2 \mathrm{um}$. This corresponds to a central average ion current density of $0.095 \mathrm{nA} / \mathrm{um}^{2}$, which is very similar to the current density at $500 \mathrm{~V}$ of a $\mathrm{Ga}^{+}$beam produced by a regular FIB column. The FWHM is easily adjustable by changing the nozzleto-sample distance, as shown in Figure 3. The influence of the electron beam current to the central millrate shows a linear behavior for beam currents up to almost $100 \mathrm{nA}$ as shown in Figure 4.

In a DualBeam the source can be used to clean up samples that have been exposed to a $\mathrm{Ga}^{+} \mathrm{FIB}$. In this way, implanted $\mathrm{Ga}$ can be removed and a possible amorphization layer can be reduced to a lower level, matching the low energy of the $\mathrm{Ar}^{+}$ions. This can be done without chamber vent. Change of gas type is fairly easy as the principle of the source is based on direct electron impact ionization: different gases have different cross-sections but there is no complex RF or DC gas discharge. Other applications of the source are the local removal of hydrocarbons from the sample, low damage deposition using gas chemistry and possible improvement of the deposition purity, when using oxygen for removal of carbon. 


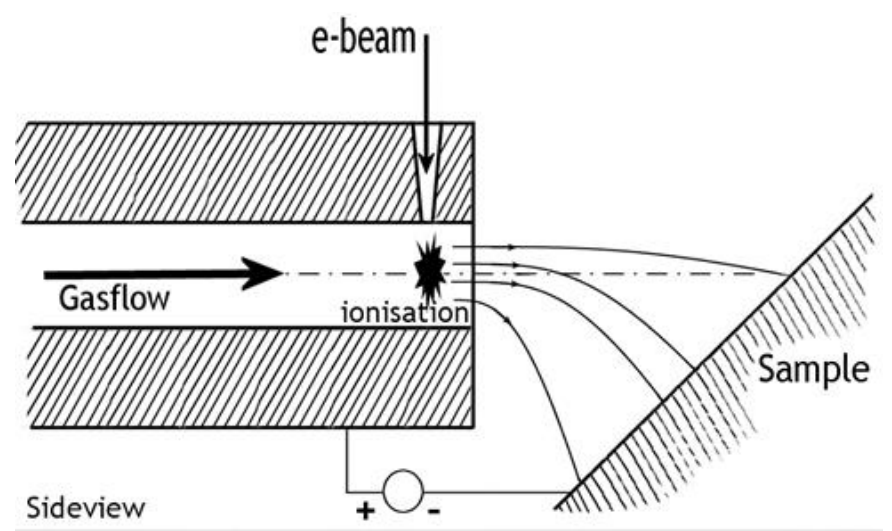

Figure 1: Schematic set up of an in-situ low energy ion source. The field defined by the distance sample-nozzle and the applied bias induces acceleration of ions towards the sample surface.

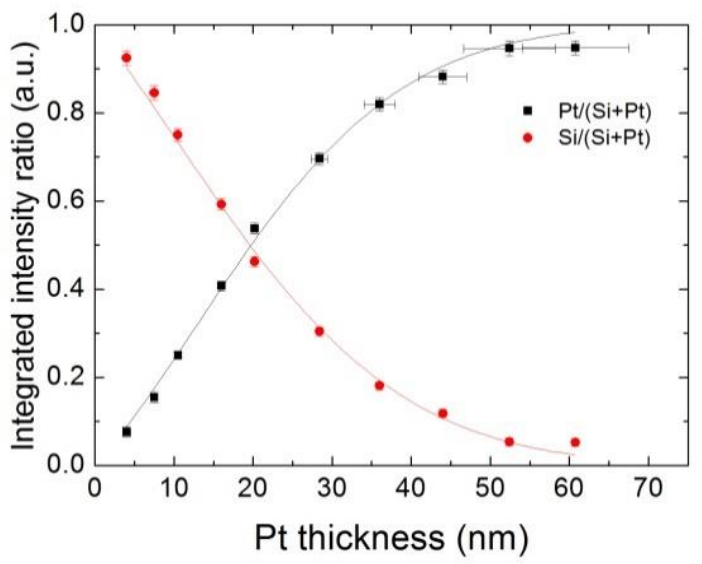

Figure 2: EDX calibration graph relating the NPI of $\mathrm{Pt}$ and $(\mathrm{Pt}+\mathrm{Si})$ to the thickness of the Pt film, for an electron beam energy of $5 \mathrm{kV}$.

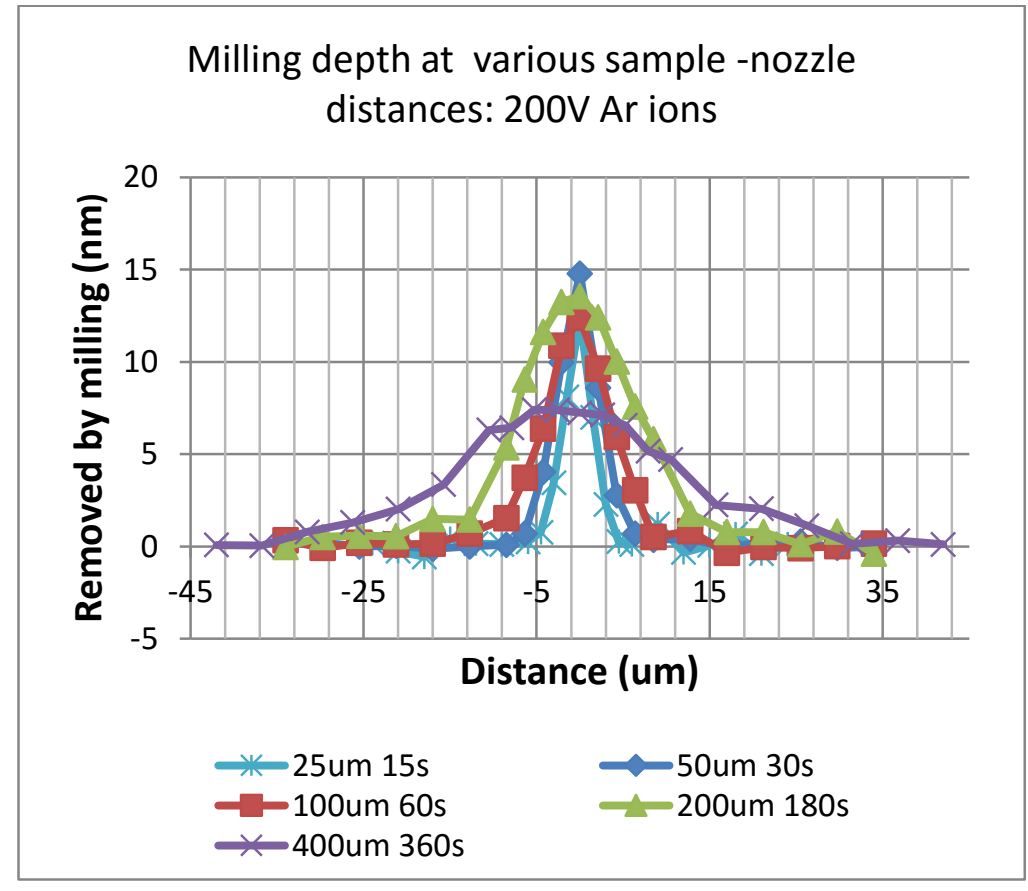

Figure 3: Beam profiles using 200 Volt bias and various gap distances. For increasing gap distances the profile gets wider, but it changes only moderately due to the slight focusing behavior of the source.

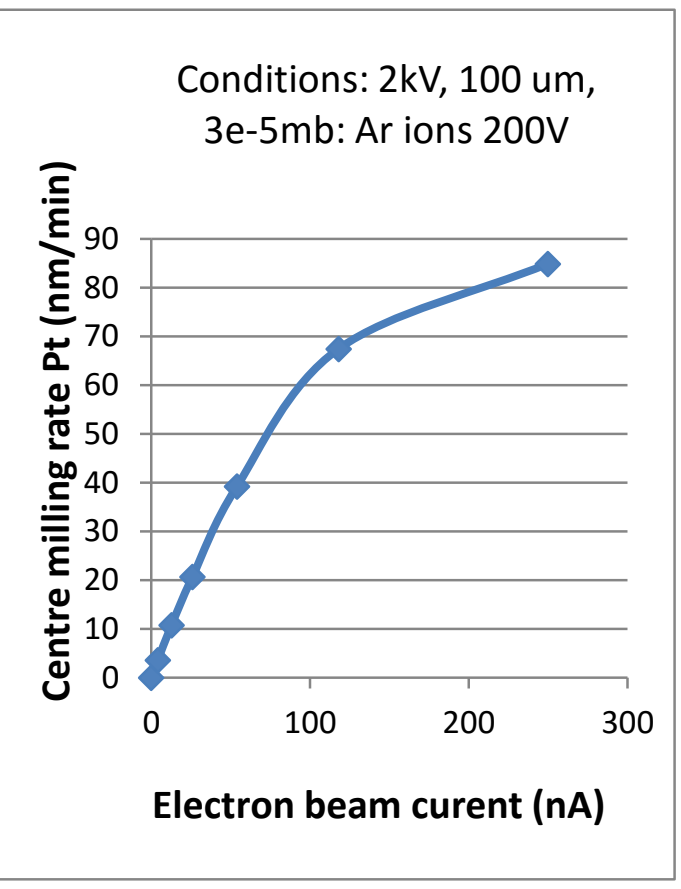

Figure 4: The center mill-rate of $\mathrm{Pt}$ as a function of the electron beam current shows an almost linear behavior up to $100 \mathrm{nA}$ 